\title{
Seleção de clones de batata para processamento industrial em condições de clima subtropical e temperado
}

\author{
Zilmar da Silva Souza(1), Dilson Antônio Bisognin(2), Glademir Roque Morin Junior(2) \\ e Francisco Saccol Gnocato(2)
}

\begin{abstract}
(1)Empresa de Pesquisa Agropecuária e Extensão Rural de Santa Catarina, Estação Experimental de São Joaquim, Caixa Postal 81, CEP 88600000 São Joaquim, SC. E mail: zilmar@epagri.sc.gov.br (2)Universidade Federal de Santa Maria, Rua Roraima, no 1.000, Camobi, CEP 97105900 Santa Maria, RS. E mail: dilsonb@smail.ufsm.br, glademirmorin@gmail.com, francisco.saccolgnocato@gmail.com
\end{abstract}

Resumo - O objetivo deste trabalho foi selecionar clones de batata para processamento industrial, cultivados em condições de clima subtropical e temperado do Sul do Brasil. Foram avaliados 30 clones avançados e três cultivares (testemunhas), na primavera de 2007 e 2008, e no outono de 2008, em Júlio de Castilhos, RS (condição de clima subtropical), e no verão de 2008 e 2009, em São Joaquim, SC (condição de clima temperado). Utilizouse o delineamento experimental de blocos ao acaso, em arranjo fatorial (33 clones em 5 ambientes), com duas ou três repetições de dez covas. Foram avaliados: a produção por cova, a aparência, a cor de chips e os teores de matéria seca e de açúcares redutores dos tubérculos. Houve interação significativa entre clones e ambientes para todas as características avaliadas, exceto quanto à cor de chips. A produção por cova e o teor de matéria seca dos tubérculos foram maiores no cultivo de verão. As condições ambientais de cultivo afetaram todos os caracteres avaliados. As condições de cultivo do Sul do Brasil possibilitam a seleção de clones de batata, com suficiente teor de matéria seca, para atender às exigências da indústria de processamento de batata.

Termos para indexação: Solanum tuberosum, açúcares redutores, cor de chips, ganho genético, melhoramento de plantas, soma de postos.

\section{Selection of potato clones for industrial processing under subtropical and temperate climate conditions}

\begin{abstract}
The objective of this work was to select potato clones for industrial processing, cultivated under subtropical and temperate conditions in Southern Brazil. Thirty advanced clones and three cultivars (control) were evaluated in the Spring of 2007 and 2008, and in the Autumn of 2008, in Júlio de Castilhos, RS (subtropical climate condition), and in the Summer of 2008 and 2009, in São Joaquim, SC (temperate climate condition). It was used a randomized complete block design in a factorial arrangement (33 clones in 5 environments), with two or three replicates of ten hill plots. Yield, appearance, chip color, dry matter and reducing sugar contents of tubers were evaluated. There was a significant interaction between clones and environments for all the evaluated traits, except for chip color. The yield and dry matter of tubers were higher in the summer crop. Environmental conditions affected all the evaluated traits. Growing conditions in Southern Brazil allow for the selection of potato clones with enough content of dry matter to meet the potato processing industry requirements.
\end{abstract}

Index terms: Solanum tuberosum, reducing sugars, chip color, selection gain, plant breeding, sum of ranks.

\section{Introdução}

No Brasil, a batata é comercializada principalmente in natura, e a quantidade destinada para processamento industrial é menor do que $10 \%$ (Popp, 2005). A procura por produtos industrializados da batata está crescendo no Brasil, pelas mudanças nos hábitos alimentares, necessidade de se obter comida semipronta, produtos mais uniformes e práticos e, também, pelo aumento das cadeias de restaurantes, o que demanda matéria prima de alta qualidade para processamento industrial.
Há requerimentos específicos quanto à qualidade dos tubérculos de batata para processamento industrial. $\mathrm{Na}$ produção de batata chips, é necessário o uso de tubérculos com formato redondo, ausência de problemas fisiológicos e, principalmente, altos teores de matéria seca (20 a 24\%) e baixos teores de açúcares redutores (10 a $15 \mathrm{mg} \mathrm{g}^{-1}$ de matéria seca) (Müller et al., 2009). $\mathrm{O}$ alto teor de matéria seca reduz a absorção de gordura durante a fritura, melhora a textura e crocância e reduz o custo de produção (Pereira, 2003), enquanto o baixo teor de açúcares redutores evita o escurecimento dos

Pesq. agropec. bras., Brasília, v.46, n.11, p.1503-1512, nov. 2011 
produtos processados que compromete a aparência, a textura e o sabor (Sowokinos, 2001; Rodrigues \& Pereira, 2003). Essas características de qualidade são determinadas pela cultivar, mas são muito influenciadas pelas condições de cultivo. No Sul do Brasil, há diferentes condições de cultivo: regiões subtropicais com dois cultivos anuais, de primavera e outono; e regiões temperadas de altitude, com um cultivo anual de verão (Epagri, 2002; Heldwein et al., 2009). Os cultivos de primavera e outono se dão em condições contrastantes de temperatura, fotoperíodo e disponibilidade de radiação solar, o que afeta a produção e a qualidade dos tubérculos para processamento industrial (Freitas et al., 2006; Bisognin et al., 2008c; Müller et al., 2009), principalmente dos clones menos adaptados. O cultivo de primavera é feito em condições que maximizam a produção e o teor de matéria seca dos tubérculos e a coloração clara de chips (Zorzella et al., 2003; Freitas et al., 2006; Pereira et al., 2007; Müller et al., 2009). As condições de outono são desfavoráveis à qualidade, principalmente em razão do acúmulo de açúcares redutores (Freitas et al., 2006; Müller et al., 2009) e do aumento do período de dormência dos tubérculos (Bisognin et al., 2008b). A radiação solar é o fator mais limitante no cultivo de outono (Bisognin et al., 2008c). Essas diferenças ambientais entre os dois cultivos, de primavera e outono, em condições de clima subtropical, fazem com que a seleção quanto às características de qualidade de tubérculo seja mais eficiente no cultivo de primavera (Müller et al., 2009).

A maioria das cultivares de batata plantadas no Brasil e do germoplasma disponível para melhoramento foi desenvolvida em regiões de clima temperado. Por isso, as condições de clima temperado de altitude do Sul do Brasil, com um cultivo anual no verão, são mais favoráveis e, portanto, possibilitam maior produção e qualidade dos tubérculos (Epagri, 2002). Dessa forma, são necessários novos estudos para avaliar o comportamento de cultivares de batata em condições de clima subtropical e temperado de cultivo, para a seleção de cultivares que combinem características de produção e qualidade de tubérculos para o processamento industrial.

O objetivo deste trabalho foi selecionar clones de batata para processamento industrial, cultivados em condições de clima subtropical e temperado de cultivo do Sul do Brasil.

\section{Material e Métodos}

Os experimentos foram realizados nos cultivos de primavera de 2007 e 2008 e de outono de 2008, em Júlio de Castilhos, RS, em condição de clima subtropical, e no cultivo de verão de 2008 e 2009, em São Joaquim, SC, em condição de clima temperado. Foram avaliados 30 clones avançados, do Programa de Melhoramento Genético de Batata, pré-selecionados em São Joaquim, SC, e três cultivares como testemunhas. Os tubérculos-sementes utilizados nos cultivos de primavera de 2007 e de verão de 2008 foram obtidos no cultivo de verão de 2007, em São Joaquim, SC. Nos demais cultivos, em Júlio de Castilhos, RS, os tubérculossementes foram tratados com $30 \mathrm{mg} \mathrm{L}^{-1}$ de ácido giberélico, para quebra da dormência, e armazenados a $20^{\circ} \mathrm{C}$. Para os cultivos de verão, em São Joaquim, SC, os tubérculos-sementes foram armazenados a $4^{\circ} \mathrm{C}$.

Utilizou-se o delineamento experimental de blocos ao acaso, em arranjo fatorial com 30 clones e três cultivares testemunhas, em cinco ambientes, com duas repetições na primavera de 2007 , no verão e outono de 2008, e três repetições na primavera de 2008 e no verão de 2009. Os clones foram avaliados em parcelas de dez covas, sem repetição e disposta em fileira única, no espaçamento de $0,30 \mathrm{~m}$, com $0,75 \mathrm{~m}$ entre as fileiras de clones. Os tratos culturais e o manejo seguiram o sistema de produção tecnificado para a cultura da batata (Epagri, 2002).

A colheita foi realizada logo após o completo secamento das plantas dos clones mais tardios e, 15 dias depois, os tubérculos foram avaliados quanto à produção por cova, aparência, cor de chips e teores de matéria seca e de açúcares redutores. A produção por cova foi medida pela razão da massa de matéria fresca de tubérculos da parcela pelo número de covas. A aparência dos tubérculos foi avaliada com base em notas visuais de 1 (pior aparência) a 5 (melhor aparência), tendo-se considerado o formato, o tamanho maior, o número de tubérculos e a ausência de defeitos fisiológicos na parcela (Bisognin \& Douches, 2002; Silva et al., 2008). A cor de chips foi determinada em dez fatias de cinco tubérculos, fritas a $185^{\circ} \mathrm{C}$, tendose atribuído notas visuais de 2 (mais claro) a 10 (mais escuro) (Bisognin \& Douches, 2002). O teor de matéria seca foi determinado em uma amostra de 150 a $250 \mathrm{~g}$, seca em estufa a $60^{\circ} \mathrm{C}$, até se obter massa constante. $\mathrm{O}$ teor de açúcares redutores foi determinado pelo método do 2,4 dinitrofenol (Freitas et al., 2006). 
Os dados foram submetidos à análise de variância, e as médias comparadas pelo teste de Scott-Knott, a $5 \%$ de probabilidade. As análises foram realizadas com auxílio do programa NTIA (Ambiente de software NTIA, 1997).

A identificação dos melhores clones foi feita com base na soma de postos (Mulamba \& Mock, 1978), que consistiu em classificar os clones em relação a cada um dos cinco caracteres, em ordem favorável ao melhoramento, e em obter a soma de postos. Os clones com soma de postos menor que a melhor testemunha foram selecionados.

\section{Resultados e Discussão}

Houve efeito significativo para ambientes e clones, quanto aos cinco caracteres avaliados, e a interação clones $\mathrm{x}$ ambientes não foi significativa somente quanto à cor de chips (Tabela 1). Nas análises individuais de cada ambiente, não foram observadas diferenças entre clones quanto ao teor de açúcares redutores e, no cultivo de primavera de 2007, quanto à produção por cova e cor de chips.

A produção por cova foi maior nos cultivos realizados em condição de clima temperado (Tabela 2), o que pode ser atribuído às temperaturas mais favoráveis, à maior disponibilidade de radiação solar e ao maior período disponível para o desenvolvimento das plantas no cultivo de verão, em razão das condições de altitude em São Joaquim, SC (Manrique \& Bartholomew, 1991). Em condições de clima temperado, com um cultivo anual, os tubérculos-sementes brotam naturalmente, ao passo que em condições de clima subtropical, com dois cultivos anuais, normalmente é necessária a quebra da dormência (Bisognin et al., 2008c), o que pode resultar em menor número de hastes por cova. Em Júlio de Castilhos, RS, o número médio de hastes por cova foi 3,3 e, em São Joaquim, foi 4,7 (Souza, 2010). O maior número de hastes também pode ter contribuído para o aumento da produção (Beukema \& Van Der Zaag, 1990).

Nos ambientes avaliados em condição de clima subtropical, a maior produção média por cova foi obtida na primavera de 2007, com tubérculossementes produzidos em condição de clima temperado e com rompimento natural da dormência (Tabela 2). Na primavera, o cultivo é realizado a temperaturas e fotoperíodo crescentes, que favorecem o desenvolvimento das plantas e a produção de tubérculos; o oposto ocorre no cultivo de outono (Andreu, 2005; Müller et al., 2009). Entretanto, no ano de 2008, a produção no cultivo de outono foi maior do que a observada na primavera. Esse resultado não era esperado, pois as condições de cultivo de outono são menos favoráveis. No entanto, alguns fatores podem explicar esse resultado no cultivo da primavera de 2008, como o atraso na emergência das plantas que ocorreu aos 33 dias na média de todos os clones, com menor período ótimo para o crescimento e desenvolvimento dos tubérculos. Isso pode afetar negativamente a produção e a qualidade dos tubérculos, pela exposição às altas temperaturas de dezembro, que resultam no aumento da taxa respiratória (Heldwein et al., 2009). As diferenças de produção observadas entre os ambientes de outono e primavera da região Sul já haviam sido relatadas em trabalhos anteriores (Rodrigues \& Pereira, 2003; Freitas et al., 2006; Bisognin et al., 2008c; Muller

Tabela 1. Resumo da análise de variância para ambientes, clones e interação ambientes x clones de 30 clones e três cultivares (testemunhas) de batata, avaliados em cinco ambientes da Região Sul do Brasil.

\begin{tabular}{|c|c|c|c|c|c|}
\hline Fonte de variação & $\begin{array}{l}\text { Tubérculos } \\
\text { por cova }(\mathrm{g})\end{array}$ & $\begin{array}{l}\text { Aparência } \\
\text { (notas 1-5) }\end{array}$ & $\begin{array}{l}\text { Cor de chips } \\
(\text { notas } 2-10)\end{array}$ & $\begin{array}{l}\text { Matéria } \\
\text { seca }(\%)\end{array}$ & $\begin{array}{c}\text { Açúcares } \\
\text { redutores }\left(\mathrm{mg} \mathrm{g}^{-1}\right)\end{array}$ \\
\hline Ambiente (A) & ** & $* *$ & $* *$ & $* *$ & $* *$ \\
\hline Primavera de 2007, Júlio de Castilhos, RS & ns & * & ns & $* *$ & ns \\
\hline Verão de 2008, São Joaquim, SC & $* *$ & $* *$ & ** & $* *$ & ns \\
\hline Outono de 2008, Júlio de Castilhos, RS & $*$ & $* *$ & $*$ & * & ns \\
\hline Primavera de 2008, Júlio de Castilhos, RS & $* *$ & $* *$ & $*$ & $* *$ & ns \\
\hline Verão de 2009, São Joaquim, SC & $* *$ & $* *$ & * & $* *$ & ns \\
\hline Clones $(\mathrm{C})$ & $* *$ & $* *$ & $* *$ & $* *$ & $* *$ \\
\hline $\mathrm{A} \times \mathrm{C}$ & $*$ & $* *$ & ns & $* *$ & $*$ \\
\hline Média & 663,9 & 3,9 & 5,3 & 22,5 & 17,3 \\
\hline $\mathrm{CV}(\%)$ & 21,8 & 13,2 & 14,6 & 5,1 & 58,4 \\
\hline
\end{tabular}

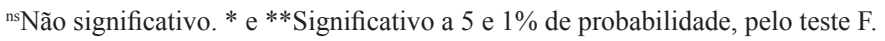


et al., 2009), porém se desconhecia a magnitude das diferenças em relação à condição de clima temperado para o cultivo. No presente trabalho, ficou evidente o efeito das diferentes condições de cultivo do Sul do Brasil, na produção de tubérculos por cova, um caráter altamente correlacionado ao rendimento da cultura da batata (Beukema \& Van Der Zaag, 1990) e, portanto, tal efeito pode ser utilizado como parâmetro de seleção de clones. Além disso, as diferentes condições de cultivo do Sul do Brasil podem ser utilizadas para maximizar o ganho genético de seleção quanto à amplitude da adaptação (Bisognin et al., 2008c).

Com relação à aparência dos tubérculos, houve significância para os fatores ambientes e clones, e interação significativa entre ambientes e clones (Tabela 2). A aparência dos tubérculos envolve vários caracteres como tamanho, embonecamento, rachaduras, deformações e outros defeitos fisiológicos muito

Tabela 2. Produção de tubérculos por cova (g) e aparência dos tubérculos (notas de 1-5) de 30 clones e três (testemunhas) de batata, nos cultivos de primavera e outono em condição de clima subtropical (Júlio de Castilhos, RS), e de verão em condição de clima temperado (São Joaquim, SC), e média de cor de chips (notas de 2-10) nos cinco ambientes ${ }^{(1)}$.

\begin{tabular}{|c|c|c|c|c|c|c|c|c|c|c|c|}
\hline \multirow[t]{3}{*}{ Clone } & \multicolumn{5}{|c|}{ Produção por cova } & \multicolumn{5}{|c|}{ Aparência dos tubérculos } & \multirow[t]{3}{*}{ Cor de chips } \\
\hline & \multicolumn{3}{|c|}{ Clima subtropical } & \multicolumn{2}{|c|}{ Clima temperado } & \multicolumn{3}{|c|}{ Clima subtropical } & \multicolumn{2}{|c|}{ Clima temperado } & \\
\hline & $\begin{array}{c}\text { Primavera } \\
2007 \\
\end{array}$ & $\begin{array}{c}\text { Outono } \\
2008\end{array}$ & $\begin{array}{c}\text { Primavera } \\
2008 \\
\end{array}$ & $\begin{array}{l}\text { Verão } \\
2008\end{array}$ & $\begin{array}{l}\text { Verão } \\
2009 \\
\end{array}$ & $\begin{array}{c}\text { Primavera } \\
2007\end{array}$ & $\begin{array}{c}\text { Outono } \\
2008\end{array}$ & $\begin{array}{c}\text { Primavera } \\
2008 \\
\end{array}$ & $\begin{array}{l}\text { Verão } \\
2008 \\
\end{array}$ & $\begin{array}{l}\text { Verão } \\
2009 \\
\end{array}$ & \\
\hline SJSM98075 1 & $587,5 \mathrm{c})$ & $345,0 \mathrm{e}$ & $451,8 \mathrm{~d}$ & $1.004,8 \mathrm{a}$ & $763,0 \mathrm{~b}$ & $4,0 \mathrm{a}$ & $4,0 \mathrm{a}$ & $4,0 \mathrm{a}$ & $3,5 \mathrm{~b}$ & $2,7 \mathrm{c}$ & $5,1 \mathrm{~b}$ \\
\hline SJSM99159 8 & $597,7 \mathrm{c}$ & $457,5 \mathrm{~d}$ & $372,5 \mathrm{e}$ & $1.010,6 \mathrm{~b}$ & $1.046,7 \mathrm{a}$ & $4,0 \mathrm{c}$ & $4,0 \mathrm{c}$ & $4,3 b$ & $4,5 \mathrm{a}$ & $3,3 \mathrm{~d}$ & $4,9 b$ \\
\hline SJSM00211 3 & $758,1 \mathrm{c}$ & $357,5 \mathrm{e}$ & $462,5 \mathrm{~d}$ & $1.034,4 \mathrm{a}$ & $843,1 b$ & $4,0 \mathrm{~d}$ & $4,5 b$ & $4,3 \mathrm{c}$ & $4,5 b$ & $5,0 \mathrm{a}$ & $5,2 \mathrm{~b}$ \\
\hline SJSM01212 1 & $445,3 \mathrm{~d}$ & $476,3 \mathrm{c}$ & $446,0 \mathrm{~d}$ & $1.065,6 \mathrm{~b}$ & $1.156,7 \mathrm{a}$ & $4,5 \mathrm{a}$ & $4,5 \mathrm{a}$ & $4,3 \mathrm{a}$ & $3,5 \mathrm{bc}$ & $4,3 \mathrm{a}$ & $5,0 \mathrm{~b}$ \\
\hline SJSM01212 2 & $515,6 \mathrm{c}$ & $519,4 d$ & $448,0 \mathrm{e}$ & $1.357,5 \mathrm{a}$ & $873,8 b$ & $4,0 \mathrm{a}$ & $4,0 \mathrm{a}$ & $4,0 \mathrm{a}$ & $4,0 \mathrm{a}$ & $4,3 \mathrm{a}$ & $5,1 \mathrm{~b}$ \\
\hline SJSM01217 8 & $783,1 \mathrm{c}$ & $750,7 \mathrm{~d}$ & $415,7 \mathrm{e}$ & $1.057,3 \mathrm{~b}$ & $1.098,7 \mathrm{a}$ & $4,0 \mathrm{a}$ & $3,0 \mathrm{~b}$ & $4,0 \mathrm{a}$ & $4,0 \mathrm{a}$ & $3,0 \mathrm{~b}$ & $5,2 \mathrm{~b}$ \\
\hline SJSM01231 10 & $603,1 b$ & $155,5 \mathrm{~d}$ & $229,3 c$ & $959,4 a$ & $957,0 \mathrm{a}$ & $4,0 \mathrm{c}$ & $4,0 \mathrm{c}$ & $3,3 \mathrm{~d}$ & $5,0 \mathrm{a}$ & $4,3 b$ & $4,8 b$ \\
\hline SJSM01250 1 & $547,6 \mathrm{c}$ & $431,5 \mathrm{e}$ & $441,0 \mathrm{~d}$ & $813,0 \mathrm{~b}$ & $909,6 \mathrm{a}$ & $4,0 \mathrm{a}$ & $4,0 \mathrm{a}$ & $4,0 \mathrm{a}$ & $4,0 \mathrm{a}$ & $3,3 b$ & $5,1 \mathrm{~b}$ \\
\hline SJSM01263 1 & $559,4 \mathrm{c}$ & $236,2 \mathrm{e}$ & $352,4 d$ & $1.093,8 \mathrm{a}$ & $943,3 b$ & $4,0 \mathrm{~d}$ & $4,5 b$ & $4,3 \mathrm{c}$ & $3,5 \mathrm{e}$ & $4,7 \mathrm{a}$ & $5,4 \mathrm{~b}$ \\
\hline SJSM01263 6 & $628,8 \mathrm{c}$ & $313,9 \mathrm{e}$ & $434,9 \mathrm{~d}$ & $1.009,4 \mathrm{a}$ & $821,7 \mathrm{~b}$ & $4,5 \mathrm{a}$ & $4,5 \mathrm{a}$ & $4,3 \mathrm{a}$ & $4,5 \mathrm{a}$ & $4,7 \mathrm{a}$ & $5,4 \mathrm{~b}$ \\
\hline SJSM01263 17 & $593,1 \mathrm{c}$ & $528,6 \mathrm{~d}$ & $488,3 \mathrm{e}$ & $906,6 \mathrm{~b}$ & $1.018,9 \mathrm{a}$ & $5,0 \mathrm{a}$ & $3,5 \mathrm{e}$ & $4,3 \mathrm{c}$ & $4,5 b$ & $3,7 \mathrm{~d}$ & $5,8 \mathrm{a}$ \\
\hline SJSM01273 1 & $643,1 \mathrm{c}$ & $305,8 \mathrm{e}$ & $482,4 d$ & $733,8 b$ & $876,7 \mathrm{a}$ & $4,0 \mathrm{a}$ & $4,0 \mathrm{a}$ & $3,3 \mathrm{c}$ & $4,0 \mathrm{a}$ & $3,7 \mathrm{~b}$ & $4,8 \mathrm{~b}$ \\
\hline SJSM01274 3 & $438,8 \mathrm{c}$ & $349,8 \mathrm{~d}$ & $277,0 \mathrm{e}$ & $849,1 \mathrm{a}$ & $660,1 b$ & $4,0 \mathrm{c}$ & $5,0 \mathrm{a}$ & $4,0 \mathrm{c}$ & $5,0 \mathrm{a}$ & $4,3 b$ & $5,0 \mathrm{~b}$ \\
\hline SJSM01274 4 & $574,4 d$ & $687,5 \mathrm{c}$ & $404,6 \mathrm{e}$ & $801,9 a$ & $770,1 b$ & $4,5 \mathrm{c}$ & $4,5 \mathrm{c}$ & $4,7 b$ & $5,0 \mathrm{a}$ & $4,3 \mathrm{~d}$ & $5,0 \mathrm{~b}$ \\
\hline SJSM01275 3 & $685,4 \mathrm{c}$ & $312,7 \mathrm{e}$ & $360,4 d$ & $1.068,8 \mathrm{a}$ & $846,7 \mathrm{~b}$ & $4,0 \mathrm{a}$ & $3,5 b$ & $4,0 \mathrm{a}$ & $3,5 \mathrm{~b}$ & $4,0 \mathrm{a}$ & $5,1 \mathrm{~b}$ \\
\hline SJSM02317 3 & $535,8 \mathrm{~d}$ & $710,7 \mathrm{c}$ & $431,7 \mathrm{e}$ & $1.218,8 \mathrm{a}$ & $895,1 \mathrm{~b}$ & $3,5 \mathrm{c}$ & $3,0 \mathrm{e}$ & $3,7 b$ & $4,0 \mathrm{a}$ & $3,3 \mathrm{~d}$ & $5,1 \mathrm{~b}$ \\
\hline SJSM02339 1 & $491,9 \mathrm{c}$ & $319,4 \mathrm{e}$ & $362,8 \mathrm{~d}$ & $896,3 a$ & $825,0 \mathrm{~b}$ & $4,0 \mathrm{a}$ & $3,5 b$ & $4,0 \mathrm{a}$ & $4,0 \mathrm{a}$ & $3,3 \mathrm{c}$ & $4,7 b$ \\
\hline SJSM02349 1 & $446,3 \mathrm{c}$ & $475,7 d$ & $369,3 \mathrm{e}$ & $1.209,4 \mathrm{a}$ & $640,0 \mathrm{~b}$ & $5,0 \mathrm{a}$ & $4,0 \mathrm{~b}$ & $3,7 \mathrm{c}$ & $5,0 \mathrm{a}$ & $3,7 \mathrm{c}$ & $5,9 \mathrm{a}$ \\
\hline SJSM02377 33 & $680,0 \mathrm{c}$ & $378,6 \mathrm{e}$ & $473,9 \mathrm{~d}$ & $1.084,4 \mathrm{a}$ & $854,3 b$ & $4,0 \mathrm{~b}$ & $4,5 \mathrm{a}$ & $4,0 \mathrm{~b}$ & $3,5 \mathrm{c}$ & $4,0 \mathrm{~b}$ & $5,3 \mathrm{~b}$ \\
\hline SJSM02382 28 & $457,5 \mathrm{c}$ & $227,3 \mathrm{e}$ & $363,5 \mathrm{~d}$ & $853,1 \mathrm{a}$ & $721,8 b$ & $4,0 \mathrm{~b}$ & $4,0 \mathrm{~b}$ & $3,3 \mathrm{~d}$ & $4,5 \mathrm{a}$ & $3,7 \mathrm{c}$ & $4,6 b$ \\
\hline SJSM03425 26 & $514,6 \mathrm{c}$ & $365,6 \mathrm{~d}$ & $296,9 \mathrm{e}$ & $1.006,3 \mathrm{a}$ & $760,6 b$ & $3,5 \mathrm{c}$ & $3,5 \mathrm{c}$ & $4,0 \mathrm{a}$ & $4,0 \mathrm{a}$ & $3,7 \mathrm{~b}$ & $4,7 b$ \\
\hline SJSM03463 1 & $634,3 \mathrm{c}$ & $501,3 \mathrm{~d}$ & $351,4 \mathrm{e}$ & $1.155,0 \mathrm{a}$ & $1.051,8 \mathrm{~b}$ & $4,0 \mathrm{~b}$ & $4,0 \mathrm{~b}$ & $4,0 \mathrm{~b}$ & $4,5 \mathrm{a}$ & $3,3 \mathrm{c}$ & $6,5 \mathrm{a}$ \\
\hline SJSM03463 3 & $563,8 \mathrm{~b}$ & $355,0 \mathrm{c}$ & $262,7 d$ & $886,9 \mathrm{a}$ & $907,5 \mathrm{a}$ & $4,0 \mathrm{~b}$ & $4,0 \mathrm{~b}$ & $4,0 \mathrm{~b}$ & $5,0 \mathrm{a}$ & $2,7 \mathrm{c}$ & $5,8 \mathrm{a}$ \\
\hline SJSM03475 31 & $606,3 \mathrm{c}$ & $362,5 \mathrm{~d}$ & $335,3 \mathrm{e}$ & $1.103,1 b$ & $1.173,3 \mathrm{a}$ & $4,0 \mathrm{~b}$ & $4,0 \mathrm{~b}$ & $4,0 \mathrm{~b}$ & $5,0 \mathrm{a}$ & $5,0 \mathrm{a}$ & $5,4 \mathrm{~b}$ \\
\hline SJSM03478 37 & $656,3 \mathrm{~d}$ & $741,9 \mathrm{c}$ & $394,9 \mathrm{e}$ & $1.203,1 \mathrm{a}$ & $936,0 \mathrm{~b}$ & $3,5 \mathrm{c}$ & $4,0 \mathrm{~b}$ & $4,3 \mathrm{a}$ & $3,0 \mathrm{e}$ & $3,3 \mathrm{~d}$ & $5,3 \mathrm{~b}$ \\
\hline SJSM04503 6 & $586,3 \mathrm{c}$ & $379,4 \mathrm{e}$ & $416,0 \mathrm{~d}$ & $1111,0 \mathrm{a}$ & $823,7 \mathrm{~b}$ & $4,0 \mathrm{~d}$ & $4,0 \mathrm{~d}$ & $4,3 \mathrm{c}$ & $4,5 \mathrm{~b}$ & $4,7 \mathrm{a}$ & $5,7 \mathrm{a}$ \\
\hline SJSM04503 9 & $550,6 \mathrm{c}$ & $618,1 b$ & $405,1 \mathrm{~d}$ & $1253,8 \mathrm{a}$ & $1.239,8 \mathrm{a}$ & $4,0 \mathrm{~d}$ & $4,0 \mathrm{~d}$ & $4,7 \mathrm{a}$ & $3,5 \mathrm{~b}$ & $2,7 \mathrm{c}$ & $5,5 \mathrm{~b}$ \\
\hline SJSM04509 9 & $688,1 \mathrm{c}$ & $378,1 \mathrm{e}$ & $384,1 d$ & $1061,3 a$ & $854,3 b$ & $4,0 \mathrm{a}$ & $4,0 \mathrm{a}$ & $4,0 \mathrm{a}$ & $3,5 \mathrm{~b}$ & $2,7 \mathrm{c}$ & $5,8 \mathrm{a}$ \\
\hline SJSM04514 2 & $703,8 \mathrm{c}$ & $367,5 \mathrm{~d}$ & $354,1 \mathrm{e}$ & $975,0 \mathrm{~b}$ & $997,7 \mathrm{a}$ & $4,0 \mathrm{a}$ & $3,0 \mathrm{~d}$ & $3,3 \mathrm{c}$ & $3,0 \mathrm{~d}$ & $3,7 \mathrm{~b}$ & $5,4 \mathrm{~b}$ \\
\hline SMA519 1 & $398,8 \mathrm{c}$ & $195,0 \mathrm{e}$ & $306,3 \mathrm{~d}$ & $745,0 \mathrm{a}$ & $584,3 \mathrm{~b}$ & $4,0 \mathrm{~d}$ & $3,5 \mathrm{e}$ & $4,3 \mathrm{c}$ & $5,0 \mathrm{a}$ & $4,7 \mathrm{~b}$ & $5,0 \mathrm{~b}$ \\
\hline 'Asterix' & $846,9 \mathrm{c}$ & $547,5 \mathrm{e}$ & $566,8 \mathrm{~d}$ & $1.223,1 \mathrm{a}$ & $1.143,3 b$ & $4,0 \mathrm{a}$ & $2,0 \mathrm{c}$ & $2,7 \mathrm{~b}$ & $2,0 \mathrm{c}$ & $2,0 \mathrm{c}$ & $6,1 \mathrm{a}$ \\
\hline 'Atlantic' & $420,6 \mathrm{e}$ & $472,5 \mathrm{~d}$ & $495,8 \mathrm{c}$ & $930,0 \mathrm{~b}$ & $1.072,1 \mathrm{a}$ & $3,5 \mathrm{c}$ & $3,5 \mathrm{c}$ & $3,7 b$ & $4,0 \mathrm{a}$ & $4,0 \mathrm{a}$ & $5,2 \mathrm{~b}$ \\
\hline 'Panda' & $484,4 \mathrm{c}$ & $270,2 \mathrm{~d}$ & $245,3 \mathrm{e}$ & $1.111,3 \mathrm{a}$ & $1.077,8 \mathrm{~b}$ & $4,0 \mathrm{~b}$ & $3,5 \mathrm{~d}$ & $3,7 \mathrm{c}$ & $4,5 \mathrm{a}$ & $3,3 \mathrm{e}$ & $5,9 \mathrm{a}$ \\
\hline Média & 582,6 & 421,0 & 390,4 & $1.024,0$ & 913,5 & 4,0 & 3,9 & 4,0 & 4,1 & 3,8 & 5,3 \\
\hline $\mathrm{CV}(\%)$ & 29,6 & 36,8 & 25,4 & 13,3 & 14,4 & 8,0 & 13,2 & 10,7 & 14,0 & 15,4 & 14,6 \\
\hline
\end{tabular}

${ }^{(1)}$ Médias seguidas de letras iguais, nas linhas quanto à produção de tubérculos por cova e aparência dos tubérculos, e, nas colunas quanto à cor de chips, não diferem entre si, pelo teste de Scott-Knott, a 5\% de probabilidade. 
afetados pelo ambiente e, em consequência, de baixa herdabilidade, enquanto a profundidade de gemas e o formato apresentam alta herdabilidade (Tai \& Yong, 1984; Andreu, 2005; Silva et al., 2008). As variações entre clones foram mais acentuadas nos cultivos do outono de 2008 e do verão de 2009. A ocorrência de geadas, antes da completa maturação das plantas no outono de 2008, e alguns períodos com deficit de umidade no solo, principalmente no cultivo de verão de 2009, foram os fatores que, possivelmente, mais afetaram a aparência dos tubérculos. Há, na literatura, relatos de que estresses por altas temperaturas e flutuações de umidade no solo afetam a aparência dos tubérculos (Kumar et al., 2004; Thomson et al., 2008).

Com relação aos valores da cor de chips, houve efeito de clones e ambientes, porém não houve interação significativa entre os fatores nem efeito significativo do fator clone no cultivo da primavera de 2007 (Tabelas 1 e 2). No cultivo de outono, os clones avaliados apresentaram cor de chips mais escura, com média de 7,3, em relação aos demais ambientes, que tiveram médias de 4,8 e 5,7 , para os cultivos de primavera em 2007 e 2008, e 4,1 e 5,6, para os cultivos de verão em 2008 e 2009, respectivamente. A cor de chips é um dos caracteres mais importantes na seleção de clones de batata para processamento industrial, e é muito influenciado pelas características genéticas e os ambientes de cultivo (Sowokinos, 2001) e altamente correlacionado aos teores de açúcares redutores (Kumar et al., 2004).

Quanto aos teores de matéria seca nos tubérculos, houve efeito significativo dos fatores ambientes e clones e interação significativa entre fatores (Tabela 3 ). Ao se analisar apenas as épocas de cultivos (ambientes), pode-se observar a tendência de maior acúmulo de matéria seca nos tubérculos produzidos em condição de clima temperado. No cultivo de verão do Sul do Brasil, as condições meteorológicas são mais favoráveis ao cultivo da batata, o que não acontece nos cultivos de outono e primavera (Beukema \& Van Der Zaag, 1990). Em cultivo na condição de clima temperado, as plantas completam o ciclo com temperatura e fotoperíodo favorável, ao passo que nos cultivos de primavera e outono, as condições meteorológicas tendem a ser mais desfavoráveis ao acúmulo de matéria seca. Os teores de matéria seca observados no cultivo da primavera de 2007 podem ser considerados baixos, em comparação aos obtidos no cultivo de outono, em condição mais desfavorável, pois o calor excessivo no final do ciclo diminuiu a fotossíntese líquida e pode ter reduzido os teores de matéria seca dos clones (Bisognin et al., 2008c; Heldwein et al., 2009). Em outros estudos de avaliação de clones e cultivares de batata para processamento industrial, realizados nos cultivos de primavera e outono no Sul do Brasil (Rodrigues \& Pereira, 2003; Freitas et al., 2006; Silva et al., 2008; Bisognin et al., 2008b; Müller et al., 2009), também observaram-se resultados similares.

Ao se considerar os limites de teores de matéria seca entre 20 e $24 \%$, para processamento industrial (Pereira, 2003), os valores obtidos na maioria dos clones e cultivares testemunhas foram adequados. Esse resultado indica que o teor de matéria seca, obtido em condições de clima subtropical e temperado do Sul do Brasil, é suficiente para atender à qualidade da matéria prima para processamento na forma de chips ou palito $\mathrm{e}$, isoladamente, não se constitui em problema para o desenvolvimento da indústria de batata processada. Entretanto, teores adequados de matéria seca deverão estar acompanhados de baixos teores de açúcares redutores, para garantir a alta qualidade dos produtos processados (Sowokinos, 2001; Kumar et al., 2004).

Quanto aos teores de açúcares redutores, houve efeito significativo dos fatores ambientes e clones, e interação significativa entre fatores. Nos ambientes individuais, não houve diferença entre clones (Tabelas 1 e 3). Os teores mais elevados foram observados no cultivo de outono, com valores acima dos aceitos em tubérculos para processamento industrial. Teores elevados de açúcares redutores na matéria prima são indesejáveis, pois promovem produtos escuros e impróprios para a comercialização. Os altos teores de açúcares redutores observados no outono estão associados às baixas temperaturas e à falta de maturidade dos tubérculos colhidos (Sowokinos, 2001), em virtude da redução da radiação solar e da ocorrência de geadas próximo ao final do ciclo das plantas, que resultaram na morte prematura da parte aérea. Além disso, baixas temperaturas e menor disponibilidade de radiação solar, característicos do cultivo de outono no Sul do Brasil (Heldwein et al., 2009), favorecem o acúmulo de açúcares redutores e o escurecimento dos chips (Kumar et al., 2004; Freitas et al., 2006; Thomson et al., 2008), como resultado da reação de Maillard.

O teor de açúcares redutores e a cor dos chips são caracteres quantitativos altamente correlacionados 
(Kumar et al., 2004) e de baixa herdabilidade (Tai \& Young, 1984). As diferenças observadas entre ambientes de cultivo, nos diferentes clones, reforça o efeito do ambiente no acúmulo de açúcares redutores (Tai \& Young, 1984; Kumar et al., 2004). Alguns clones avaliados podem ser considerados promissores pelos baixos teores de açúcares redutores, observados nos cultivos de primavera e verão, nos quais se consideram os teores desejáveis entre 10 e
$15 \mathrm{mg} \mathrm{g}^{-1}$, para o processamento na forma de chips (Freitas et al., 2006; Müller et al., 2009). Entretanto, pelos resultados observados, o cultivo de outono não deve ser indicado para seleção. Assim, a seleção de clones quanto aos teores de açúcares redutores deve ser realizada apenas nos cultivos que melhor expressam a variabilidade genética existente (Bisognin et al., 2008a); neste caso, nos cultivos de primavera e verão. Os resultados obtidos no presente trabalho, acrescentados

Tabela 3. Teores de matéria seca (\%) e de açúcares redutores $\left(\mathrm{mg} \mathrm{g}^{-1}\right)$ de 30 clones de três cultivares (testemunhas) de batata, nos cultivos de primavera e outono em condição de clima subtropical (Júlio de Castilhos, RS), e de verão em condição de clima temperado (São Joaquim, SC) ${ }^{(1)}$.

\begin{tabular}{|c|c|c|c|c|c|c|c|c|c|c|}
\hline \multirow[t]{3}{*}{ Clone } & \multicolumn{5}{|c|}{ Teor de matéria seca } & \multicolumn{5}{|c|}{ Teor de açúcares redutores } \\
\hline & \multicolumn{3}{|c|}{ Clima subtropical } & \multicolumn{2}{|c|}{ Clima temperado } & \multicolumn{3}{|c|}{ Clima subtropical } & \multicolumn{2}{|c|}{ Clima temperado } \\
\hline & $\begin{array}{c}\text { Primavera } \\
2007 \\
\end{array}$ & $\begin{array}{c}\text { Outono } \\
2008 \\
\end{array}$ & $\begin{array}{c}\text { Primavera } \\
2008 \\
\end{array}$ & $\begin{array}{l}\text { Verão } \\
2008 \\
\end{array}$ & $\begin{array}{l}\text { Verão } \\
2009 \\
\end{array}$ & $\begin{array}{c}\text { Primavera } \\
2007 \\
\end{array}$ & $\begin{array}{c}\text { Outono } \\
2008 \\
\end{array}$ & $\begin{array}{c}\text { Primavera } \\
2008 \\
\end{array}$ & $\begin{array}{l}\text { Verão } \\
2008 \\
\end{array}$ & $\begin{array}{l}\text { Verão } \\
2009 \\
\end{array}$ \\
\hline $\begin{array}{l}\text { SJSM98075 } 1 \\
\end{array}$ & $20,8 \mathrm{e}$ & $20,9 \mathrm{c}$ & $20,8 \mathrm{~d}$ & $23,8 \mathrm{a}$ & $23,0 \mathrm{~b}$ & $11,7 \mathrm{~d}$ & $25,5 b$ & $20,3 c$ & $52,4 a$ & $10,1 \mathrm{e}$ \\
\hline SJSM99159 8 & $21,4 d$ & $21,3 \mathrm{e}$ & $24,8 b$ & $24,4 \mathrm{c}$ & $25,1 \mathrm{a}$ & $27,4 b$ & $33,4 \mathrm{a}$ & $23,4 \mathrm{c}$ & $22,0 \mathrm{~d}$ & $10,6 \mathrm{e}$ \\
\hline SJSM00211 3 & $22,4 d$ & $22,2 \mathrm{e}$ & $22,5 \mathrm{c}$ & $24,0 \mathrm{a}$ & $23,8 b$ & 6,1 & $34,4 \mathrm{a}$ & $23,1 b$ & $21,8 \mathrm{c}$ & $9,6 \mathrm{~d}$ \\
\hline SJSM01212 1 & $18,4 d$ & $20,6 c$ & $22,6 b$ & $23,0 \mathrm{~b}$ & $24,4 \mathrm{a}$ & $8,9 b$ & $24,9 \mathrm{a}$ & $10,6 \mathrm{~b}$ & $11,6 \mathrm{~b}$ & $7,8 \mathrm{~b}$ \\
\hline SJSM01212 2 & $20,5 \mathrm{c}$ & $22,3 b$ & $23,7 \mathrm{a}$ & $23,2 \mathrm{a}$ & $22,8 b$ & $7,8 \mathrm{~b}$ & $29,3 \mathrm{a}$ & $12,7 \mathrm{~b}$ & $13,2 b$ & $12,2 \mathrm{~b}$ \\
\hline SJSM01217 8 & $20,5 \mathrm{e}$ & $22,2 d$ & $23,2 \mathrm{c}$ & $25,2 \mathrm{a}$ & $24,4 \mathrm{~b}$ & $7,9 \mathrm{~d}$ & $39,9 a$ & $13,4 \mathrm{~b}$ & $7,6 \mathrm{~d}$ & $9,4 \mathrm{c}$ \\
\hline SJSM01231 10 & $21,3 \mathrm{~d}$ & $20,5 \mathrm{e}$ & $23,0 \mathrm{c}$ & $23,2 b$ & $23,7 \mathrm{a}$ & $5,3 \mathrm{e}$ & $55,3 \mathrm{a}$ & $11,4 \mathrm{c}$ & $14,6 \mathrm{~b}$ & $10,7 \mathrm{~d}$ \\
\hline SJSM01250 1 & $23,8 b$ & $23,3 \mathrm{c}$ & $24,7 \mathrm{a}$ & $24,5 \mathrm{a}$ & $24,4 \mathrm{a}$ & $6,1 d$ & $38,3 \mathrm{a}$ & $10,5 \mathrm{c}$ & $25,6 \mathrm{~b}$ & $12,4 \mathrm{c}$ \\
\hline SJSM01263 1 & $21,2 b$ & $19,8 \mathrm{~b}$ & $22,3 a$ & $23,1 \mathrm{a}$ & $20,8 b$ & $7,3 b$ & $41,0 \mathrm{a}$ & $12,1 b$ & $17,4 \mathrm{~b}$ & $9,6 \mathrm{~b}$ \\
\hline SJSM01263 6 & $19,4 b$ & $20,7 b$ & $22,0 \mathrm{a}$ & $22,4 \mathrm{a}$ & $20,6 b$ & $8,9 b$ & $32,4 a$ & $6,4 b$ & $16,6 \mathrm{~b}$ & $10,8 b$ \\
\hline SJSM01263 17 & $21,2 \mathrm{c}$ & $20,5 d$ & $23,3 \mathrm{a}$ & $22,8 b$ & $23,2 \mathrm{a}$ & $23,3 b$ & $52,6 \mathrm{a}$ & $17,5 \mathrm{c}$ & $13,0 \mathrm{~d}$ & $10,6 \mathrm{e}$ \\
\hline SJSM01273 1 & $22,6 b$ & $23,7 \mathrm{a}$ & $23,8 b$ & $25,1 \mathrm{a}$ & $23,7 b$ & $16,0 \mathrm{~b}$ & $15,9 b$ & $19,9 \mathrm{a}$ & 16,5 & $15,7 b$ \\
\hline SJSM01274 3 & $19,3 \mathrm{e}$ & $22,9 b$ & $21,4 d$ & $24,3 \mathrm{a}$ & $21,8 \mathrm{c}$ & $10,5 \mathrm{~d}$ & $32,4 a$ & $12,6 \mathrm{c}$ & $16,8 \mathrm{~b}$ & $10,0 \mathrm{~d}$ \\
\hline SJSM01274 4 & $24,8 \mathrm{c}$ & $22,5 \mathrm{e}$ & $22,9 d$ & $25,5 b$ & $26,8 \mathrm{a}$ & $4,7 \mathrm{~d}$ & $25,1 \mathrm{a}$ & $9,0 \mathrm{c}$ & $8,3 c$ & $11,5 b$ \\
\hline SJSM01275 3 & $21,7 \mathrm{c}$ & $22,1 b$ & $21,4 \mathrm{c}$ & $23,4 \mathrm{a}$ & $22,3 b$ & $6,3 c$ & $43,4 \mathrm{a}$ & $8,1 \mathrm{c}$ & $14,6 \mathrm{~b}$ & $15,4 b$ \\
\hline SJSM02317 3 & $19,4 \mathrm{e}$ & $23,2 d$ & $24,4 b$ & $23,7 \mathrm{c}$ & $25,6 \mathrm{a}$ & $41,5 \mathrm{a}$ & $23,4 b$ & $9,2 \mathrm{e}$ & $19,1 \mathrm{c}$ & $10,0 \mathrm{~d}$ \\
\hline SJSM02339 1 & $18,2 \mathrm{e}$ & $21,4 d$ & $23,2 b$ & $22,2 \mathrm{c}$ & $26,3 \mathrm{a}$ & $24,8 b$ & $46,0 \mathrm{a}$ & $10,8 \mathrm{e}$ & $14,0 \mathrm{c}$ & $12,9 \mathrm{~d}$ \\
\hline SJSM02349 1 & $19,8 \mathrm{e}$ & $20,8 \mathrm{~d}$ & $22,4 b$ & $23,2 \mathrm{a}$ & $21,8 \mathrm{c}$ & $5,3 \mathrm{e}$ & $25,5 \mathrm{a}$ & $12,5 \mathrm{c}$ & $6,6 \mathrm{~d}$ & $13,0 \mathrm{~b}$ \\
\hline SJSM02377 33 & $22,9 d$ & $22,6 \mathrm{e}$ & $23,0 \mathrm{c}$ & $25,5 \mathrm{a}$ & $23,5 b$ & $4,9 \mathrm{e}$ & $67,1 \mathrm{a}$ & $9,7 \mathrm{~d}$ & $12,1 \mathrm{~b}$ & $10,2 \mathrm{c}$ \\
\hline SJSM02382 28 & $22,1 \mathrm{c}$ & $21,8 \mathrm{e}$ & $22,0 b$ & $24,0 \mathrm{a}$ & $21,9 \mathrm{~d}$ & $15,4 \mathrm{~b}$ & $50,8 \mathrm{a}$ & $11,7 \mathrm{~d}$ & $14,6 \mathrm{c}$ & $9,8 \mathrm{e}$ \\
\hline SJSM03425 26 & $19,6 \mathrm{e}$ & $22,9 \mathrm{c}$ & $21,2 \mathrm{~d}$ & $24,0 \mathrm{~b}$ & $25,0 \mathrm{a}$ & $6,6 \mathrm{e}$ & $36,5 \mathrm{a}$ & $12,9 \mathrm{~d}$ & $14,6 \mathrm{c}$ & $16,2 b$ \\
\hline SJSM03463 1 & $19,8 \mathrm{e}$ & $21,1 d$ & $21,8 \mathrm{c}$ & $21,8 b$ & $23,6 \mathrm{a}$ & $23,9 b$ & $45,3 \mathrm{a}$ & $13,4 d$ & $10,8 \mathrm{e}$ & $17,5 \mathrm{c}$ \\
\hline SJSM03463 3 & $18,2 d$ & $21,4 \mathrm{c}$ & $18,1 \mathrm{c}$ & $23,0 \mathrm{a}$ & $22,8 \mathrm{~b}$ & $18,8 \mathrm{~d}$ & $49,4 \mathrm{a}$ & $19,8 \mathrm{c}$ & $39,3 b$ & $16,3 \mathrm{e}$ \\
\hline SJSM03475 31 & $17,1 \mathrm{e}$ & $19,9 d$ & $20,2 \mathrm{c}$ & $21,5 \mathrm{a}$ & $21,2 b$ & $7,6 \mathrm{~d}$ & $28,8 \mathrm{a}$ & $7,9 \mathrm{c}$ & $6,3 \mathrm{e}$ & $11,6 b$ \\
\hline SJSM03478 37 & $23,8 \mathrm{c}$ & $22,0 \mathrm{e}$ & $23,3 d$ & $25,9 b$ & $27,2 \mathrm{a}$ & $12,1 \mathrm{c}$ & $40,3 \mathrm{a}$ & $8,1 \mathrm{e}$ & $10,0 \mathrm{~d}$ & $16,7 b$ \\
\hline SJSM04503 6 & $22,5 b$ & $22,0 \mathrm{~d}$ & $22,4 \mathrm{e}$ & $24,8 \mathrm{a}$ & $22,2 \mathrm{c}$ & $8,4 \mathrm{e}$ & $31,6 b$ & $18,4 \mathrm{c}$ & $37,5 \mathrm{a}$ & $10,6 \mathrm{~d}$ \\
\hline SJSM045039 & $18,7 \mathrm{e}$ & $22,0 \mathrm{c}$ & $21,3 d$ & $22,9 b$ & $24,1 \mathrm{a}$ & $13,1 \mathrm{c}$ & $26,6 a$ & $7,5 \mathrm{e}$ & $13,6 b$ & $10,5 \mathrm{~d}$ \\
\hline SJSM04509 9 & $18,5 \mathrm{e}$ & $20,1 d$ & $20,7 \mathrm{c}$ & $22,5 \mathrm{a}$ & $20,9 b$ & $8,3 d$ & $39,0 \mathrm{a}$ & $4,4 \mathrm{e}$ & $14,7 \mathrm{~b}$ & $13,3 \mathrm{c}$ \\
\hline SJSM04514 2 & $20,5 \mathrm{~d}$ & $23,2 b$ & $20,7 \mathrm{c}$ & $24,2 \mathrm{a}$ & $20,2 \mathrm{e}$ & $14,8 \mathrm{e}$ & $54,9 \mathrm{a}$ & $18,4 \mathrm{c}$ & $23,2 b$ & $14,9 \mathrm{~d}$ \\
\hline SMA519 1 & $24,2 \mathrm{~d}$ & $21,3 \mathrm{e}$ & $24,3 \mathrm{c}$ & $26,1 \mathrm{a}$ & $25,8 \mathrm{~b}$ & $8,3 \mathrm{c}$ & $20,8 \mathrm{a}$ & $7,8 \mathrm{e}$ & $14,4 \mathrm{~b}$ & $8,2 \mathrm{~d}$ \\
\hline 'Asterix ' & $19,8 \mathrm{e}$ & $20,3 d$ & $21,8 \mathrm{c}$ & $22,8 \mathrm{a}$ & $22,7 b$ & $13,0 \mathrm{~d}$ & $28,4 a$ & $28,3 b$ & $19,6 \mathrm{c}$ & $11,5 \mathrm{e}$ \\
\hline 'Atlantic' & $21,1 \mathrm{e}$ & $22,5 \mathrm{~d}$ & $24,0 \mathrm{~b}$ & $24,2 \mathrm{a}$ & $23,9 \mathrm{c}$ & $8,5 \mathrm{e}$ & $20,4 a$ & $12,0 \mathrm{c}$ & $18,7 \mathrm{~b}$ & $10,2 d$ \\
\hline 'Panda' & $22,6 \mathrm{e}$ & $23,2 d$ & $23,9 \mathrm{c}$ & $24,5 \mathrm{a}$ & $24,0 \mathrm{~b}$ & $6,0 \mathrm{e}$ & $29,0 \mathrm{a}$ & $19,3 b$ & $15,3 \mathrm{~d}$ & $15,7 \mathrm{c}$ \\
\hline Média & 20,9 & 21,7 & 22,4 & 23,8 & 23,4 & 12,1 & 36,0 & 13,4 & 17,5 & 12,0 \\
\hline $\mathrm{CV}(\%)$ & 5,4 & 5,2 & 6,3 & 2,1 & 4,7 & 74,6 & 39,4 & 70,2 & 84,4 & 38,6 \\
\hline
\end{tabular}

${ }^{(1)}$ Médias seguidas de letras iguais, nas linhas, não diferem entre si, pelo teste de Scott-Knott, a 5\% de probabilidade. 
aos de outros já realizados no Rio Grande do Sul (Rodrigues \& Pereira, 2003; Freitas et al., 2006; Pereira et al., 2007; Bisognin et al., 2008b; Müller et al., 2009), ratificam que o cultivo de outono é pouco favorável para a produção de tubérculos para atender à indústria de processamento.

A identificação dos melhores clones foi baseada na soma de postos dos cinco caracteres avaliados, em comparação à melhor testemunha (Tabela 4). Assim, os clones selecionados foram os que apresentaram o melhor equilíbrio de atributos favoráveis para a produção e qualidade dos tubérculos para processamento industrial. Na média dos ambientes, foram selecionados 11 clones considerados de ampla adaptação. Em condição de clima subtropical, foram selecionados sete clones e, em condição de clima temperado, foram selecionados 16 clones. Ao se compararem os clones selecionados, apenas cinco (SJSM01212 2, SJSM01274 4, SJSM99159 8, SJSM00211 3 e SJSM03478 37) foram selecionados e considerados de ampla adaptação, tanto

Tabela 4. Índice e soma de postos, conforme Mulamba \& Mock (1978), de 30 clones e 3 cultivares testemunhas de batata, quanto à produção por cova (PC), aparência dos tubérculos (AT), notas de cor de chips (CC) e teores de matéria seca (MS) e de açúcares redutores (AR), nos cultivos de primavera de 2007 e 2008 e outono de 2008, em Júlio de Castilhos, RS (condição de clima subtropical), e de verão de 2008 e 2009, em São Joaquim, SC (condição de clima temperado), e na média dos cinco ambientes.

\begin{tabular}{|c|c|c|c|c|c|c|c|c|c|c|c|c|c|c|c|c|c|c|}
\hline \multirow[t]{2}{*}{ Clone } & \multicolumn{6}{|c|}{ Júlio de Castilhos, RS (clima subtropical) } & \multicolumn{6}{|c|}{ São Joaquim, SC (clima temperado) } & \multicolumn{6}{|c|}{ Média dos ambientes } \\
\hline & $\mathrm{PC}$ & AT & $\mathrm{CC}$ & MS & $\mathrm{AR}$ & Soma & $\mathrm{PC}$ & AT & $\mathrm{CC}$ & MS & $\mathrm{AR}$ & Soma & $\mathrm{PC}$ & AT & $\mathrm{CC}$ & MS & $\mathrm{AR}$ & Soma \\
\hline$\overline{\text { SJSM01263 } 1}$ & 28 & 5 & 20 & 22 & 19 & 94 & 12 & 12 & 22 & 30 & 16 & 92 & 22 & 11 & 21 & 29 & 17 & 100 \\
\hline SJSM01263 6 & 20 & 2 & 21 & 27 & 8 & 78 & 23 & 7 & 23 & 32 & 17 & 102 & 20 & 2 & 23 & 30 & 8 & 83 \\
\hline SJSM01212 $1^{(1)}$ & 21 & 3 & 17 & 29 & 6 & 76 & 5 & 19 & 3 & 15 & 3 & 45 & 8 & 10 & 8 & 23 & 5 & 54 \\
\hline SJSM01212 2(2) & 11 & 13 & 13 & 12 & 9 & 58 & 4 & 11 & 14 & 21 & 12 & 62 & 6 & 14 & 14 & 15 & 9 & 58 \\
\hline SJSM01275 3 & 22 & 22 & 8 & 16 & 17 & 85 & 20 & 23 & 16 & 25 & 22 & 106 & 18 & 21 & 11 & 20 & 18 & 88 \\
\hline SJSM01273 $1^{(3)}$ & 13 & 24 & 4 & 3 & 11 & 55 & 29 & 20 & 4 & 10 & 27 & 90 & 26 & 22 & 4 & 5 & 13 & 70 \\
\hline SJSM01250 $1^{(4)}$ & 16 & 14 & 7 & 1 & 13 & 51 & 27 & 25 & 17 & 9 & 29 & 107 & 24 & 20 & 12 & 4 & 19 & 79 \\
\hline SJSM01263 17(5) & 6 & 6 & 23 & 17 & 33 & 85 & 17 & 13 & 31 & 22 & 8 & 91 & 10 & 12 & 29 & 19 & 30 & 100 \\
\hline SJSM01274 $3^{(5)}$ & 29 & 4 & 14 & 20 & 14 & 81 & 32 & 4 & 6 & 20 & 14 & 76 & 32 & 3 & 9 & 21 & 12 & 77 \\
\hline SJSM01274 $4^{(2)}$ & 5 & 1 & 9 & 2 & 2 & 19 & 31 & 5 & 12 & 2 & 5 & 55 & 19 & 1 & 10 & 1 & 1 & 32 \\
\hline SJSM01217 $8^{(1)}$ & 2 & 28 & 11 & 14 & 21 & 76 & 8 & 28 & 18 & 4 & 1 & 59 & 2 & 30 & 17 & 11 & 10 & 70 \\
\hline SJSM01231 10 & 32 & 25 & 2 & 18 & 24 & 101 & 18 & 6 & 8 & 18 & 11 & 61 & 29 & 13 & 5 & 17 & 21 & 85 \\
\hline SJSM98075 1 & 18 & 15 & 18 & 26 & 16 & 93 & 25 & 31 & 13 & 19 & 33 & 121 & 23 & 27 & 15 & 22 & 31 & 118 \\
\hline SJSM99159 $8^{(2)}$ & 14 & 11 & 5 & 8 & 30 & 68 & 11 & 16 & 9 & 6 & 28 & 70 & 11 & 15 & 6 & 8 & 29 & 69 \\
\hline SJSM00211 $3^{(2)}$ & 7 & 7 & 22 & 10 & 22 & 68 & 21 & 3 & 7 & 13 & 26 & 70 & 13 & 4 & 18 & 12 & 20 & 67 \\
\hline SJSM04509 9 & 12 & 16 & 30 & 31 & 10 & 99 & 19 & 32 & 27 & 31 & 18 & 127 & 16 & 28 & 27 & 32 & 11 & 114 \\
\hline SJSM04514 2 & 15 & 31 & 25 & 19 & 31 & 121 & 14 & 29 & 19 & 29 & 30 & 121 & 14 & 32 & 24 & 24 & 32 & 126 \\
\hline SJSM04503 6 & 19 & 12 & 28 & 15 & 18 & 92 & 16 & 8 & 24 & 16 & 31 & 95 & 17 & 6 & 26 & 13 & 26 & 88 \\
\hline SJSM04503 $9^{(1)}$ & 8 & 8 & 24 & 30 & 7 & 77 & 1 & 9 & 25 & 17 & 9 & 61 & 3 & 7 & 25 & 25 & 7 & 67 \\
\hline SJSM03475 31(1) & 23 & 17 & 15 & 33 & 5 & 93 & 3 & 1 & 29 & 33 & 2 & 68 & 9 & 5 & 22 & 33 & 3 & 72 \\
\hline SJSM03425 26(5) & 26 & 29 & 3 & 21 & 15 & 94 & 26 & 21 & 2 & 8 & 23 & 80 & 28 & 25 & 2 & 14 & 15 & 84 \\
\hline SJSM03478 37(2) & 3 & 20 & 19 & 6 & 20 & 68 & 9 & 30 & 20 & 1 & 13 & 73 & 4 & 29 & 19 & 2 & 16 & 70 \\
\hline SJSM03463 1 & 10 & 18 & 31 & 24 & 29 & 112 & 6 & 17 & 33 & 27 & 19 & 102 & 7 & 17 & 33 & 26 & 28 & 111 \\
\hline SJSM03463 3 & 25 & 19 & 29 & 32 & 32 & 137 & 24 & 22 & 28 & 24 & 32 & 130 & 27 & 18 & 28 & 31 & 33 & 137 \\
\hline SJSM02382 28(5) & 30 & 26 & 6 & 13 & 26 & 101 & 30 & 14 & 1 & 23 & 10 & 78 & 31 & 19 & 1 & 16 & 23 & 90 \\
\hline SJSM02377 33(5) & 9 & 10 & 26 & 7 & 28 & 80 & 15 & 24 & 10 & 7 & 6 & 62 & 12 & 16 & 20 & 7 & 25 & 80 \\
\hline SJSM02317 $3^{(5)}$ & 4 & 32 & 12 & 11 & 25 & 84 & 10 & 26 & 15 & 5 & 21 & 77 & 5 & 31 & 13 & 9 & 24 & 82 \\
\hline SJSM02339 1 & 27 & 23 & 1 & 25 & 27 & 103 & 28 & 27 & 11 & 12 & 15 & 93 & 30 & 24 & 3 & 18 & 27 & 102 \\
\hline SJSM02349 1 & 24 & 9 & 27 & 23 & 4 & 87 & 22 & 10 & 30 & 28 & 4 & 94 & 25 & 9 & 30 & 27 & 4 & 95 \\
\hline$\underline{\text { SMA519 }} 1^{(1)}$ & 33 & 21 & 16 & 5 & 1 & 76 & 33 & 2 & 5 & 3 & 7 & 50 & 33 & 8 & 7 & 3 & 2 & 53 \\
\hline 'Asterix' & 1 & 33 & 32 & 28 & 23 & 117 & 2 & 33 & 32 & 26 & 25 & 118 & 1 & 33 & 32 & 28 & 22 & 116 \\
\hline 'Atlantic' & 17 & 30 & 10 & 9 & 3 & 69 & 13 & 15 & 21 & 14 & 20 & 83 & 15 & 26 & 16 & 10 & 6 & 73 \\
\hline 'Panda' & 31 & 27 & 33 & 4 & 12 & 107 & 7 & 18 & 26 & 11 & 24 & 86 & 21 & 23 & 31 & 6 & 14 & 95 \\
\hline
\end{tabular}

${ }^{(1)}$ Clones selecionados em condições de clima temperado e na média dos ambientes. ${ }^{(2)}$ Clones selecionados nas condições de clima subtropical e temperado e, também, na média dos ambientes. ${ }^{(3)}$ Clone selecionado em condição de clima subtropical e na média dos ambientes. ${ }^{(4)}$ Clone selecionado em condição de clima subtropical. ${ }^{(5)}$ Clone selecionado em condição de clima temperado. Foram selecionados os clones com soma de postos menor do que a melhor testemunha (cultivar Atlantic). 
pela média dos cinco ambientes, como pela condição de climas subtropical e temperado de cultivo.

Os ganhos indiretos de seleção foram favoráveis ao melhoramento de todos os caracteres e ambientes (Tabela 5). O ganho indireto de seleção total foi similar nas três situações estudadas. O teor de açúcares redutores foi o caráter com maior destaque na média dos ambientes e em condição de clima temperado. A produção de tubérculos por cova foi mais importante na condição de clima subtropical de cultivo, seguido do teor de açúcares redutores. No entanto, o menor ganho indireto de seleção para produção por cova foi observado em condições de clima temperado, o que já era esperado, pois os clones já haviam sido preliminarmente selecionados em São Joaquim, SC.

A grande influência do ambiente sobre as características de qualidade é um desafio para o melhoramento genético da batata, principalmente em condições de clima subtropical de cultivo. A seleção de clones superiores pressupõe a reunião de vários caracteres de qualidade - geralmente quantitativos e de baixa herdabilidade -, com a produção, de forma equilibrada, em um mesmo clone (Andreu, 2005; Bisognin et al., 2008a). Outro aspecto importante a ser considerado é a necessidade de se desenvolverem clones adaptados para dois cultivos anuais, ou seja, completar o ciclo de desenvolvimento das plantas em aproximadamente 90 dias, com curta dormência, alta produção e teores adequados de matéria seca e açúcares redutores. A seleção desses clones deve ser associada a fatores fisiológicos de resposta fotoperiódica para o início da tuberização e para a rápida emergência, e aumento da área foliar para o melhor aproveitamento da radiação solar disponível (Bisognin et al., 2008c).

Ao se considerar que o germoplasma de batata utilizado está adaptado às condições de países de clima temperado, esses resultados da seleção de clones com atributos de produção, qualidade de processamento e ampla adaptação se constituem como importante avanço científico para o desenvolvimento de novas cultivares, para atender às exigências do segmento da batata processada no Brasil. O desenvolvimento inicial

Tabela 5. Média dos clones originais e dos selecionados e ganho indireto de seleção quanto à produção e à qualidade de processamento de tubérculos de batata, nos cultivos de primavera de 2007 e 2008 e outono de 2008, em Júlio de Castilhos, RS (condição de clima subtropical), e de verão de 2007 e 2008 em São Joaquim, SC (condição de clima temperado), e na média dos cinco ambientes.

\begin{tabular}{|c|c|c|c|c|}
\hline Caráter & $\begin{array}{c}\text { Média dos } \\
\text { clones originais }\end{array}$ & $\begin{array}{c}\text { Média dos } \\
\text { clones selecionados }^{(1)}\end{array}$ & $\begin{array}{c}\text { Ganho indireto } \\
\text { de seleção }\end{array}$ & $\begin{array}{l}\text { Ganho indireto } \\
\text { de seleção (\%) }\end{array}$ \\
\hline & \multicolumn{4}{|c|}{ Júlio de Castilhos, RS (Clima subtropical) } \\
\hline Produção por cova $(\mathrm{g})$ & 462,8 & 514,3 & 51,456 & 11,118 \\
\hline Aparência dos tubérculos (notas de 1-5) & 4,0 & 4,1 & 0,079 & 1,956 \\
\hline Cor de chips (notas de $2-10$ ) & 5,6 & 5,4 & 0,169 & 3,046 \\
\hline Teor de massa de matéria seca (MS) (\%) & 21,6 & 22,9 & 1,328 & 6,144 \\
\hline Teor de açúcares redutores $\left(\mathrm{mg} \mathrm{g}^{-1} \mathrm{MS}\right)$ & 20,7 & 19,2 & 1,511 & 7,291 \\
\hline \multirow[t]{2}{*}{ Total } & - & - & - & 29,555 \\
\hline & \multicolumn{4}{|c|}{ São Joaquim, SC (Clima temperado) } \\
\hline Produção por cova $(\mathrm{g})$ & 956,3 & 974,2 & 17,887 & 1,870 \\
\hline Aparência dos tubérculos (notas de 1-5) & 4,0 & 4,2 & 0,160 & 3,966 \\
\hline Cor de chips (notas de $2-10$ ) & 4,8 & 4,5 & 0,283 & 5,871 \\
\hline Teor de massa de matéria seca (MS) (\%) & 23,6 & 24,2 & 0,569 & 2,409 \\
\hline Teor de açúcares redutores $\left(\mathrm{mg} \mathrm{g}^{-1} \mathrm{MS}\right)$ & 14,7 & 12,4 & 2,327 & 15,844 \\
\hline \multirow[t]{2}{*}{ Total } & - & - & - & 29,960 \\
\hline & \multicolumn{4}{|c|}{ Médias dos ambientes } \\
\hline Produção por cova $(\mathrm{g})$ & 660,2 & 698,9 & 38,688 & 5,860 \\
\hline Aparência dos tubérculos (notas de 1-5) & 4,0 & 4,1 & 0,107 & 2,659 \\
\hline Cor de chips (notas de $2-10$ ) & 5,3 & 5,1 & 0,136 & 2,581 \\
\hline Teor de massa de matéria seca (MS) (\%) & 22,4 & 23,0 & 0,531 & 2,367 \\
\hline Teor de açúcares redutores ( $\left.\mathrm{mg} \mathrm{g}^{-1} \mathrm{MS}\right)$ & 18,3 & 15,5 & 2,826 & 15,435 \\
\hline Total & - & - & - & 28,902 \\
\hline
\end{tabular}

${ }^{(1)}$ Foram selecionados 7, 16 e 11 clones em condições de clima subtropical, clima temperado e na média dos ambientes, respectivamente. 
de clones, em condição de clima temperado de altitude, no Sul do Brasil, como em São Joaquim, SC, favorece a identificação de clones superiores que seriam, então, selecionados em condições de cultivo de primavera e outono. As regiões com clima temperado de altitude possuem características intermediárias entre clima temperado típico e de subtrópico. Este diferencial pode se constituir em importante estratégia para programa de melhoramento genético de batata no Brasil.

\section{Conclusões}

1. As condições de cultivo de outono, no Sul do Brasil, são menos favoráveis para a seleção de clones de batata, quanto à qualidade para processamento industrial dos tubérculos.

2. O teor de açúcares redutores é o caráter mais limitante na seleção de clones de batata para processamento industrial dos tubérculos.

3. As condições de cultivo do Sul do Brasil possibilitam a seleção de clones de batata com suficiente teor de matéria seca, para atender à indústria de processamento de batata.

\section{Referências}

AMBIENTE de software NTIA: manual do usuário. Versão 4.2.2. Campinas: Embrapa CNPTIA, 1997. 258p.

ANDREU, M.A. Associação entre características agronômicas da batata nos plantios de primavera e outono no Rio Grande do Sul. Ciência e Agrotecnologia, v.29, p.925-929, 2005.

BEUKEMA, H.P.; VAN DER ZAAG, D.E. Introduction to potato production. Wageningen: PUDOC, 1990. 207p.

BISOGNIN, D.A.; COSTA, L.C. da; ANDRIOLO, J.L.; MÜLLER, D.R.; BANDINELLI, M.G. Produtividade e qualidade de tubérculos de clones de batata. Ciência e Natura, v.30, p.43-56, 2008a.

BISOGNIN, D.A.; DOUCHES, D.S. Early generation selection for potato tuber quality in progenies of late blight resistant parents. Euphytica, v.127, p.1-9, 2002.

BISOGNIN, D.A.; FREITAS, S.T. de; BRACKMANN, A.; ANDRIOLO, J.L.; PEREIRA, E.I.P.; MÜLLER, D.R.; BANDINELLI, M.G. Envelhecimento fisiológico de tubérculos de batata produzidos durante o outono e a primavera e armazenados em diferentes temperaturas. Bragantia, v.67, p.59-65, $2008 \mathrm{~b}$.

BISOGNIN, D.A.; MÜLLER, D.R.; STRECK, N.A.; ANDRIOLO, J.L.; SAUSEN, D. Desenvolvimento e rendimento de clones de batata na primavera e no outono. Pesquisa Agropecuária Brasileira, v.43, p.699-705, 2008c.

EPAGRI. Sistemas de produção para batata consumo e batatasemente em Santa Catarina. 3.ed. rev. atual. Florianópolis: EPAGRI, 2002. 123p. (Epagri. Sistemas de produção, 2).
FREITAS, S.T. de; BISOGNIN, D.A.; GÓMEZ,A.C.S.; SAUTTER, C.K.; COSTA, L.C. da; RAMPELOTTO, M.V. Qualidade para processamento de clones de batata cultivados durante a primavera e o outono no Rio Grande do Sul. Ciência Rural, v.36, p.80-85, 2006.

HELDWEIN, A.B.; STRECK, N.A.; BISOGNIN, D.A. Batata. In: MONTEIRO, J.E.B.A. (Ed.). Agrometeorologia dos cultivos: o fator meteorológico na produção agrícola. Brasília: Instituto Nacional de Meteorologia, 2009. p.91-109.

KUMAR, D.; SINGH, B.P.; KUMAR, P. An overwiew of the factors affecting sugar content of potatoes. Annals of Applied Biology, v.145, p.247-256, 2004.

MANRIQUE, L.A.; BARTHOLOMEW, D.P. Growth and yield performance of potato grown at three elevations in Hawaii: II. Dry matter production and efficiency of partitioning. Crop Science, v.31, p.367-372, 1991.

MULAMBA, N.N.; MOCK, J.J. Improvement of yield potential of the Eto Blanco maize (Zea mays L.) population by breeding for plant traits. Egyptian Journal of Genetics and Citology, v.7, p.40-51, 1978.

MÜLLER, D.R.; BISOGNIN, D.A.; ANDRIOLO, J.L.; MORIN JUNIOR, G.R.; GNOCATO, F.S. Expressão dos caracteres e seleção de clones de batata nas condições de cultivo de primavera e outono. Ciência Rural, v.39, p.1237-1334, 2009.

PEREIRA, A. da S. Melhoramento genético. In: PEREIRA, A. da S.; DANIELS, J. (Ed.). O cultivo da batata na Região Sul do Brasil. Pelotas: Embrapa Clima Temperado; Brasília: Embrapa Informação Tecnológica, 2003. p.105-124.

PEREIRA,A.da S.; FRITSCHENETO, R.; SILVA, R. da S.;BENDER, C.I.; SCHÜNEMANN, A.P.; FERRI, N.M.L.; VENDRUSCOLO, J.L. Genótipos de batata com baixo teor de açúcares redutores. Horticultura Brasileira, v.25, p.220-223, 2007.

POPP, P. Processamento de batata: variedades de aptidão industrial. In: SEMINÁRIO MINEIRO SOBRE PROCESSAMENTO DE BATATAS, 2005, Pouso Alegre. Anais. Pouso Alegre: EPAMIG, 2005. 7p.

RODRIGUES, A.F.S.; PEREIRA, A. da S. Correlações inter e intragerações e herdabilidade de cor do chips, matéria seca e produção em batata. Pesquisa Agropecuária Brasileira, v.38, p.599-604, 2003.

SOUZA, Z. da S. Melhoramento da batata para processamento industrial em condições subtropical e temperada do Sul do Brasil. 2010. 151p. Tese (Doutorado) - Universidade Federal de Santa Maria, Santa Maria.

SILVA, G.O. da; PEREIRA, A. da S.; SOUZA, V.Q. de; CARVALHO, F.I.F. de; FRITSCHE NETO, R. Seleção para caracteres fenotípicos de tubérculos nas primeiras gerações em batata. Ceres, v.55, p.168-172, 2008.

SOWOKINOS, J.R. Biochemical and molecular control of cold induced sweetening in potatoes. American Journal of Potato Research, v.78, p.221-236, 2001.

TAI, G.C.C.; YOUNG, D.A. Early generation selection for important agronomic characteristics in a potato breeding population. American Potato Journal, v.61, p.419-434, 1984. 
THOMSOM,A.L.; LOVE, S.L.; SOWOKINOS, J.R.;THORNTON, M.K.; SHOCK, C.C. Review of the sugar end disorder in potato (Solanum tuberosum, L.). American Journal of Potato Research, v.85, p.375-386, 2008.
ZORZELLA, C.A.; TREPTOW, R.O.; ALMEIDA, T.L. de; VENDRUSCOLO, J.L.S. Caracterização física, química e sensorial de genótipos de batata processados na forma de chips. Brazilian Journal of Food Technology, v.6, p.15-24, 2003.

Recebido em 1ำ de novembro de 2010 e aprovado em 10 de novembro de 2011 